\title{
A Possible Role for Malonyl-CoA in the Regulation of Hepatic Fatty Acid Oxidation and Ketogenesis
}

\author{
J. Denis McGarry, Guy P. Mannaerts, and Daniel W. Foster
}

From the Departments of Internal Medicine and Biochemistry, University of Texas Health Science

Center at Dallas, Dallas, Texas 75235

A B S T RACT Studies on the oxidation of oleic and octanoic acids to ketone bodies were carried out in homogenates and in mitochondrial fractions of livers taken from fed and fasted rats. Malonyl-CoA inhibited ketogenesis from the former but not from the latter substrate. The site of inhibition appeared to be the carnitine acyltransferase I reaction. The effect was specific and easily reversible. Inhibitory concentrations were in the range of values obtained in livers from fed rats by others. It is proposed that malonylCoA functions as both precursor for fatty acid synthesis and suppressor of fatty acid oxidation. As such, it might be an important element in the carbohydrate-induced sparing of fatty acid oxidation.

\section{INTRODUCTION}

Previous studies from this laboratory have suggested that the marked enhancement of long-chain fatty acid oxidation and ketogenesis characteristic of livers from ketotic animals results from activation of the first step specific to fatty acid oxidation, namely the carnitine acyltransferase reaction (see 1 for review). The mechanism for this activation is not understood, though elevation of liver carnitine levels appears to be one component of the control system (2). It has long been known that a reciprocal relationship exists between liver glycogen content and fatty acid oxidation $(3,4)$, and we have shown that high carnitine concentrations are not sufficient to induce ketosis if hepatic glycogen content is high (5). An attractive hypothesis was that some factor related to hepatic carbohydrate metabolism served to suppress fatty acid oxidation in the fed state.

The studies to be reported below suggest that the putative suppressor intermediate is related to carbo-

J. D. McGarry is the recipient of Research Career Development Award 1-KO4-AM0763. G. P. Mannaerts was the recipient of Fogarty International Fellowship 1-F05TW223401.

Received for publication 21 April 1977. hydrate metabolism but in fact is not part of the immediate sequence of glycogen to pyruvate. Rather, it is the first intermediate specific to the fatty acid biosynthetic pathway, malonyl-CoA. Moreover, it is shown that malonyl-CoA exerts its effect by inhibiting carnitine acyltransferase I. Thus, malonyl-CoA might represent the regulatory factor through which the carbohydrate sparing of hepatic fatty acid oxidation is expressed.

\section{METHODS}

Animals. Male Sprague-Dawley rats weighing 100-150 g were used. They were fed a diet containing $58.5 \%$ sucrose, $21 \%$ casein, and less than $1 \%$ fat by weight, together with all necessary vitamins and minerals (Teklad Test Diets, Life Sci. Div., The Mogul Corp., Madison, Wis.) and used for experiments between 7:00 and 8:30 a.m. Fasted rats were deprived of food for $24 \mathrm{~h}$ before use.

Preparation of liver homogenates. Rats were killed by cervical dislocation followed by exsanguination. Liver homogenates were prepared in a modified Krebs-Henseleit bicarbonate buffer, pH 7.4, containing no calcium, twice the normal amount of $\mathrm{MgSO}_{4}, 4 \mathrm{vol}$ of $0.154 \mathrm{M} \mathrm{NaCl}$, and 100 vol of $0.154 \mathrm{M} \mathrm{KCl}$ per $130 \mathrm{vol}$ of buffer (referred to hereafter as potassium-KHB buffer). The tissue was first minced with scissors and homogenized in a Dounce homogenizer (Kontes Co., Vineland, N. J.) with six strokes each of a loose and a tight-fitting plunger. Homogenates from fed rats were brought to a final concentration of $5 \%$ (wt/vol) while those from fasted animals were prepared in a concentration of $3.1 \%$ (wt/vol). The latter dilution was used to provide concentrations of mitochondria equivalent to those obtained in the fed homogenates (judged by measurements of glutamate dehydrogenase activity in freeze-thawed preparations). The observed increase in mitochondrial content of homogenates from fasted rats presumably resulted from shrinkage in liver size after $24 \mathrm{~h}$ of starvation (35-40\%). For certain experiments mitochondrial fractions were prepared by centrifugation of the whole homogenate at $2,000 \mathrm{~g}$ for $10 \mathrm{~min}$ in a refrigerated centrifuge, removal of the supernate, and resuspension of the pellet to the original volume with potassium-KHB.

Mitochondrial integrity was evident from the following observations: (a) centrifugation of homogenates followed by freeze thawing of supernates and pellets in liquid $\mathbf{N}_{2}$ showed that $95-100 \%$ of the glutamate dehydrogenase 
and citrate synthase activities were confined to the latter fraction; (b) in the presence of ATP and coenzyme A (CoASH) oxidation of oleate was absolutely dependent upon the addition of carnitine, whereas oxidation of octanoate and L-octanoylcarnitine required neither CoASH nor carnitine; (c) the high rates of fatty acid oxidation seen in whole homogenates and mitochondrial fractions were almost completely lost upon freeze thawing in liquid $\mathrm{N}_{2} ;(d)$ respiratory control ratios expected for intact mitochondria were observed.

Experiments with homogenates. Incubations were carried out in 25-ml Erlenmeyer flasks which initially contained, in a total volume of $2.0 \mathrm{ml}$ of potassium-KHB buffer, the $\left.{ }^{[14} \mathrm{C}\right]$-labeled fatty acid and other components listed under Results. Reactions were initiated by addition of $0.5 \mathrm{ml}$ of liver homogenate or mitochondrial fraction, the flasks were gassed with 95\% $\mathrm{O}_{2}: 5 \% \mathrm{CO}_{2}$, capped with rubber stoppers from which were suspended plastic center-wells (Kontes Co.), and shaken at $37^{\circ} \mathrm{C}$ with $80-90$ oscillations per min. At the indicated times $0.4 \mathrm{ml}$ of $1 \mathrm{M}$ hyamine hydroxide in methanol and $0.4 \mathrm{ml}$ of $5 \%(\mathrm{wt} / \mathrm{vol}) \mathrm{HClO}_{4}$ were injected into the center wells and flasks, respectively. The flasks were shaken for a further $45 \mathrm{~min}$ at room temperature to trap ${ }^{14} \mathrm{CO}_{2}$ after which the center wells were placed in plastic vials and assayed for radioactivity in a liquid scintillation counter. The flask contents were transferred to tubes containing $0.3 \mathrm{ml}$ of $70 \%$ (wt/vol) $\mathrm{HClO}_{4}$ and, after centrifugation, the supernatant fraction was neutralized with $5 \mathrm{NKOH}$. The neutralized extracts were analyzed for acetoacetate and $\beta$-hydroxybutyrate and for the ${ }^{14} \mathrm{C}$ content of the individual ketone bodies as described previously (6). Throughout the text the term "ketones" will always refer to the sum of acetoacetate plus $\beta$-hydroxybutyrate.

Materials. Labeled fatty acids were from Amersham/ Searle Corp., Arlington Heights, Ill. L-carnitine was obtained from Grand Island Biological Co., Grand Island, N. Y. ATP, CoASH, acetyl-CoA, propionyl-CoA, methylmalonylCoA, and malonyl-CoA were purchased from P-L Biochemicals, Inc., Milwaukee, Wis. The sources of all other materials have been given previously $(2,5,6)$.

\section{RESULTS}

For purposes of this discussion the important steps in the hepatic oxidation of long-chain fatty acids to ketone bodies are summarized below:

(a) Fatty acid + ATP

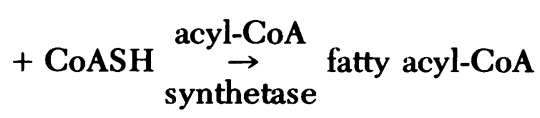$$
\text { + AMP + pyrophosphate; }
$$

(b) Fatty acyl-CoA

$$
+ \text { carnitine } \stackrel{\text { CAT I }}{\rightleftharpoons} \text { fatty acylcarnitine + CoASH; }
$$

(c) Fatty acylcarnitine

$$
+\mathrm{CoASH} \stackrel{\text { CAT II }}{\rightleftharpoons} \text { fatty acyl-CoA + carnitine; }
$$

(d) Fatty acyl-CoA $\stackrel{\beta \text {-oxidation }}{\rightarrow}$ acetyl-CoA

$$
\underset{\text { cycle }}{\rightarrow \text { HMG-CoA }} \text { ketone bodies; }
$$

where CAT I and CAT II represent carnitine acyltransferases I (located on the outer aspect of the inner mitochondrial membrane) and II (located on the inner aspect of the inner mitochondrial membrane), respectively (7).

Determination of optimal conditions for the oxidation of fatty acids by whole liver homogenates. The standard reaction mixture ultimately employed in studies with oleic acid contained in a final volume of $2.5 \mathrm{ml}$ potassium-KHB buffer:sodium $\left[1-{ }^{14} \mathrm{C}\right]$-oleate, $0.1 \mathrm{mM}$, bound to $0.8 \%$ defatted albumin (molar ratio fatty acid:albumin =0.95); ATP, $4 \mathrm{mM}$; L-carnitine, $0.1 \mathrm{mM}$; CoASH, $0.05 \mathrm{mM}$; and liver homogenate in the quantities indicated in legends to Tables. Similar conditions were used to examine the oxidation of $\left[1-{ }^{14} \mathrm{C}\right]$ octanoate, except that albumin, carnitine, and CoASH were omitted and the substrate concentration was increased to $0.2 \mathrm{mM}$. These conditions yielded oxidation rates that were maximal and linear over a period of $15 \mathrm{~min}$. Ketone bodies, mainly in the form of acetoacetate, were virtually the sole products of oleate oxidation. Essentially no endogenous fatty acid oxidation occurred under these conditions. The quantity of fatty acids oxidized through the tricarboxylic acid cycle was less than $1 \%$ of that converted into ketone bodies.

Comparison of rates of oxidation of oleate and octanoate by liver homogenates. Table I shows that both oleic acid and octanoic acid supported equally high rates of ketogenesis in homogenates from fed and fasted rats. In the case of the medium-chain fatty acid this was expected since neither CAT I nor CAT II is required for entry into the $\beta$-oxidation sequence and octanoate has previously been shown to be oxidized at similar rates in intact livers from fed, fasted, and diabetic rats (8). The rate of oleate oxidation, which is low in perfused livers from fed

\section{TABLE I}

Effects of Malonyl-CoA on the Oxidation of $\left[1-{ }^{14} \mathrm{C}\right]-O l e a t e$ and $\left[1-{ }^{14} \mathrm{C}\right]$-Octanoate by Liver Homogenates from Fed and Fasted Rats

\begin{tabular}{llcc}
\hline \multirow{2}{*}{$\begin{array}{c}\text { State of } \\
\text { animal }\end{array}$} & Malonyl-CoA & \multicolumn{2}{c}{ Fatty acid $\rightarrow$ ketones } \\
\cline { 3 - 4 } & $m M$ & \multicolumn{2}{c}{ nmol/12 min } \\
\hline \multirow{2}{*}{ Fed } & 0 & 116 & 326 \\
& 0.2 & 14 & 330 \\
Fasted & 0 & 129 & 329 \\
& 0.2 & 41 & 344
\end{tabular}

$0.5 \mathrm{ml}$ of whole liver homogenates was incubated with the standard reaction mixture described in the text. 
rats compared with those from fasted animals (9), was equally rapid in homogenates prepared from such livers. Thus, under the conditions of these experiments, the overall capacity for long-chain fatty acid oxidation in homogenates appeared to be unaltered by fasting, an observation consistent with those made by DiMarco and Hoppel with rat liver mitochondria (10).

Effect of malonyl-CoA on the oxidation of fatty acids by liver homogenates. If long-chain fatty acid oxidation were normally suppressed in the fed state by a specific factor, the high rates of ketogenesis from oleate seen in the fed homogenate might have been due to the 100-fold dilution of cellular components in the final incubation mixtures. The putative factor might logically have been sought either in the glycolytic sequence (glycogen $\rightarrow$ pyruvate) or in the fatty acid biosynthetic pathway per se (acetyl$\mathrm{CoA} \rightarrow$ fatty acids). In view of the known reciprocal relationship between rates of fatty acid synthesis and oxidation in liver, we favored the latter possibility. Since malonyl-CoA represents the first compound specific for fatty acid biosynthesis, it seemed a logical candidate. When tested in the standard incubation system at a concentration of $0.2 \mathrm{mM}$, malonyl-CoA proved to be strongly inhibitory towards oleate oxidation in homogenates from both fed and fasted rats, though the latter appeared to be somewhat less sensitive (Table I). However, the oxidation of octanoate was totally unaffected by malonyl-CoA, indicating that inhibition of oleate oxidation was exerted at a point before the $\beta$-oxidation sequence of enzymes. This might have been either the acyl-CoA synthetase step or one of the CAT reactions. That the former was

\section{TABLE II}

Effects of Various CoASH Derivatives on Oleate Oxidation by the Mitochondrial Fraction from the Liver of a Fed Rat

\begin{tabular}{lc}
\hline \multicolumn{1}{c}{ Additions } & {$\left[1-{ }^{4} \mathrm{C}\right]$ oleate $\rightarrow$ ketones } \\
\hline & $\%$ of control \\
None & 100 \\
Acetyl-CoA & 95.3 \\
Propionyl-CoA & 95.0 \\
Methylmalonyl-CoA & 101.2 \\
Malonyl-CoA & 14.5 \\
Malonyl-CoA + carnitine & 18.2 \\
\hline
\end{tabular}

$0.5 \mathrm{ml}$ of mitochondrial fraction prepared from the liver of a fed rat (see Methods) was incubated for $12 \mathrm{~min}$ with the standard reaction mixture described in the text. The absolute rate of conversion of oleate into ketones in the control incubation was $85 \mathrm{nmol} / 12 \mathrm{~min}$. The CoASH derivatives were present in a concentration of $0.2 \mathrm{mM}$. The carnitine mentioned in line 6 represents an additional quantity of $0.25 \mathrm{mM}$.

\section{TABLE III}

Effect of Malonyl-CoA on Oleylcarnitine Formation from Oleate by the Mitochondrial Fraction from the Liver of a Fed Rat

\begin{tabular}{lc}
\hline \multicolumn{1}{c}{ Additions } & Oleylcarnitine concentration \\
\hline & $\mu M$ \\
None & 4.48 \\
Carnitine $(0.25 \mathrm{mM})$ & 6.72 \\
Malonyl-CoA $(0.2 \mathrm{mM})$ & 0.23 \\
Malonyl-CoA + carnitine & 0.42 \\
\hline
\end{tabular}

Incubation conditions were as described in Table II with the exception that unlabeled oleate and $\mathrm{D}, \mathrm{L}-\left[\right.$ methyl $-{ }^{14} \mathrm{C}$ ] carnitine were used. After $6 \mathrm{~min}$, reactions were terminated with $\mathrm{HCl}$, the oleylcarnitine was extracted into butanol (11), and its ${ }^{14} \mathrm{C}$ content was determined by liquid scintillation counting.

not the affected site was established by the observation that the inhibition of oleate oxidation caused by malonyl-CoA was accompanied by a marked increase in the incorporation of the fatty acid into phospholipids and triglycerides with no change in the quantity of oleic acid utilized (data not shown). This suggested that the site of malonyl-CoA inhibition was at the level of CAT.

The data of Table II illustrate specificity for the malonyl-CoA inhibition of oleate oxidation as acetyl-CoA, propionyl-CoA, and methylmalonyl-CoA were all without significant effect. Since this experiment was performed with the mitochondrial fraction as opposed to the whole liver homogenate, it is evident that cytosolic factors are not required for expression of malonyl-CoA inhibition. However, the remote possibility existed that deacylase activity in the mitochondrial preparation preferentially hydrolyzed malonyl-CoA with the release of free CoASH and that the latter was the cause of the inhibitory effect. When an extra $200 \mu \mathrm{M}$ CoASH was added a $50 \%$ inhibition of oleate oxidation was indeed observed (experiments not shown). However, this inhibition was largely relieved by the addition of extra carnitine, suggesting that at high concentrations CoASH inhibits fatty acid oxidation by reversal of the CAT I reaction via mass action. In contrast, the inhibitory effect of malonyl-CoA was not reversed by raising the carnitine concentration, indicating a direct inhibition of either CAT I or CAT II. Since malonyl-CoA would not be expected to gain access to CAT II, which is situated on the inner aspect of the inner mitochondrial membrane (7), and since it did not inhibit the oxidation of octanoylcarnitine (data not shown), the more likely site of interaction would appear to be at the CAT I step. Direct evidence to this effect was obtained in the studies of Table 


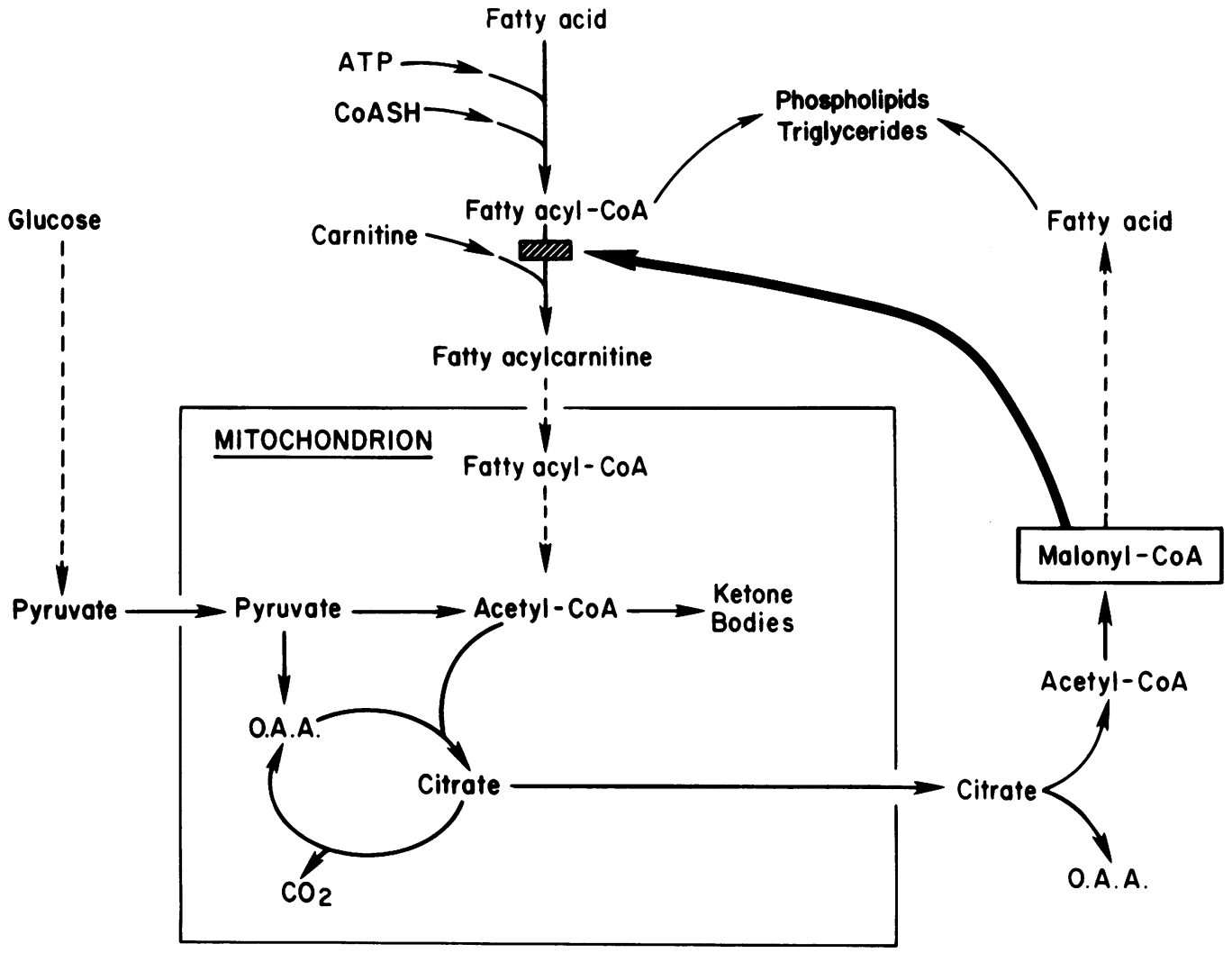

FIGURE 1 Interrelations between fatty acid biosynthesis, fatty acid oxidation, and ketogenesis in liver. O.A.A. stands for oxaloacetate.

III. In these experiments the ${ }^{14} \mathrm{C}$ label was present on the carnitine molecule rather than on the oleic acid, thus allowing determination of the oleylcarnitine concentration reached during the various incubations. In the control incubation, oleylcarnitine concentration at $6 \mathrm{~min}$ was $4.48 \mu \mathrm{M}$ and increased to $6.72 \mu \mathrm{M}$ with the further addition of $250 \mu \mathrm{M}$ carnitine. ${ }^{1} \mathrm{By}$ contrast, the acylcarnitine concentration in the presence of malonyl-CoA was reduced to $0.23 \mu \mathrm{M}$ and was not significantly altered by the addition of extra carnitine. In experiments not shown, it was established that the inhibition seen with malonyl-CoA was largely lost by prior alkaline hydrolysis and that the slight residual inhibitory effect could be fully accounted for by the amount of free CoASH released. Malonate had no inhibitory effect.

The reversibility of the malonyl-CoA inhibition of oleate oxidation was demonstrated as follows. When malonyl-CoA was added to a portion of a whole homogenate such that its final concentration in the

${ }^{1}$ Excess carnitine increased the oleylcarnitine concentration but did not affect the rate of fatty acid oxidation, indicating that at high carnitine concentrations ketogenesis becomes limited by the capacity of the $\beta$-oxidation pathway. reaction flask was $0.17 \mathrm{mM}$, oxidation of oleate was inhibited by almost $90 \%$. However, when the mitochondrial fractions were separated from both the control homogenate and that containing added malonyl-CoA, they oxidized the fatty acid at similar rates that approximated those given by the unfractionated control homogenate (data not shown).

The effect of other metabolites on oleate oxidation by rat liver homogenates. Because of the possibility mentioned earlier that intermediates in the conversion of glycogen to pyruvate might also be potential inhibitors of fatty acid oxidation, the following compounds were tested: glycogen, amylopectin, amylose, UDP-glucose, glucose, fructose, glucose-6phosphate, fructose-1,6-diphosphate, dihydroxyacetone, $s n$-glycerol-3-phosphate, 3-phosphoglycerate, 2phosphoglycerate, phosphoenolpyruvate, pyruvate, and lactate $+\mathrm{NAD}^{+}$. In addition, a series of compounds related to the pentose phosphate pathway and the tricarboxylic acid cycle were tested: 6-phosphogluconate, ribulose-5-phosphate, ribose-5-phosphate, xylulose-5-phosphate, oxaloacetate, citrate, $\alpha$-ketoglutarate, succinate, malate, alanine, aspartate, glutamate, and glutamine. None was found to have a significant effect. 


\section{DISCUSSION}

The notion that carbohydrate in some manner exerts a sparing effect on hepatic fatty acid oxidation and ketogenesis was established many years ago (12). For example, the ingestion of a small amount of glucose reverses starvation ketosis in man and inhibits the oxidation of fatty acids to ketone bodies in animals. Moreover, a reciprocal relationship exists between glycogen content of the liver and its ketogenic capacity $(4,13)$. However, the mechanism of these interactions has remained an enigma. The concept that opposing anabolic and catabolic pathways are regulated such that stimulation of one pathway is associated with the automatic suppression of the other is well established in the case of carbohydrate metabolism. For example, the processes of glycogenolysis and glycogen synthesis are usually not simultaneously active, nor are the glycolytic and gluconeogenic sequences. In both cases unidirectional carbon flow is assured through the actions of specific metabolites or cofactors. We felt that an analogous mechanism might be operative in the regulation of fatty acid synthesis and degradation. For this reason we decided to test the effects of malonyl-CoA, the first compound specific to fatty acid biosynthesis, for its ability to suppress fatty acid oxidation in liver homogenates. Malonyl-CoA was found to potently inhibit ketone body production from oleate, an effect that was not duplicated either by other CoASH derivatives or by numerous intermediates involved in glycogen-pyruvate interconversions, the pentose phosphate pathway, or the tricarboxylic acid cycle. The inhibition was shown to be reversible and to be exerted at the first step specific to long-chain fatty acid oxidation, the carnitine acyltransferase I reaction. Thus, it is the only physiological metabolite known to inhibit this enzyme.

In experiments not shown, significant inhibition was observed at concentrations of malonyl-CoA as low as $0.02 \mathrm{mM}$, a level similar to that found in fed rats by Guynn et al. (14). ${ }^{2}$ (The same authors showed that the concentration of malonyl-CoA fell fourfold during fasting.) We presume that the high and equivalent rates of oleate oxidation seen in homogenates from fed and fasted animals were due to dilution of the malonyl-CoA to noninhibitory levels in both preparations.

Illustration of the possible role of malonyl-CoA in the control of hepatic ketogenesis is given in Fig. 1. It is proposed that with carbohydrate feeding fatty acid synthetic rates are brisk, reflecting high rates of

\footnotetext{
${ }^{2}$ In a typical experiment the rate of oleate oxidation in a homogenate from fed liver was $80 \mathrm{nmol} / 10 \mathrm{~min}$. Inhibition by malonyl-CoA was: $0.02 \mathrm{mM}(27 \%), 0.05 \mathrm{mM}(77 \%), 0.1 \mathrm{mM}$ (89\%), and $0.2 \mathrm{mM}(93 \%)$.
}

malonyl-CoA generation. In these circumstances malonyl-CoA serves a dual role: substrate for fat synthesis and repressor of fat oxidation. By contrast, in starvation and diabetes, the flow of glucose carbon to fatty acids is restricted and malonyl-CoA levels are diminished with the result that fatty acid oxidation and ketone body production increase.

We have previously suggested that the activation of hepatic ketogenic capacity is brought about by elevation of the circulating glucagon to insulin ratio (13). One consequence of this hormonal imbalance is an increase in hepatic carnitine concentration which serves to drive the carnitine acyltransferase I reaction (2). We propose that a second effect is depletion of hepatic malonyl-CoA consequent to a block in the generation of pyruvate. The latter is likely due both to dissipation of liver glycogen stores as free glucose and to inhibition of the pyruvate kinase reaction (15). In this regard it is of interest that Cook et al. (16) have recently demonstrated that glucagon acutely lowers malonyl-CoA concentrations in livers from intact rats and in isolated hepatocytes.

\section{ACKNOWLEDGMENT}

The expert technical help of Martha Bennett and Petra Contreras is gratefully acknowledged.

This work was supported by U. S. Public Health Service grant AM-18573 and training grant CA 05200.

\section{REFERENCES}

1. McGarry, J. D., and D. W. Foster. 1977. Hormonal control of ketogenesis. Biochemical considerations. Arch. Intern. Med. 137: 495-501.

2. McGarry, J. D., C. Robles-Valdes, and D. W. Foster. 1975. Role of carnitine in hepatic ketogenesis. Proc. Natl. Acad. Sci. U. S. A. 72: 4385-4388.

3. Mirsky, I. A. 1942. The etiology of diabetic acidosis. J.A.M.A. (J. Am. Med. Assoc.). 118: 690-694.

4. McGarry, J. D., J. M. Meier, and D. W. Foster. 1973. The effects of starvation and refeeding on carbohydrate and lipid metabolism in vivo and in the perfused rat liver. The relationship between fatty acid oxidation and esterification in the regulation of ketogenesis. J. Biol. Chem. 248: 270-278.

5. Robles-Valdes, C., J. D. McGarry, and D. W. Foster. 1976. Maternal-fetal carnitine relationships and neonatal ketosis in the rat. J. Biol. Chem. 251: 6007-6012.

6. McGarry, J. D., and D. W. Foster. 1970. Ketone body metabolism in the ketosis of starvation and alloxan diabetes. J. Biol. Chem. 245: 4382-4390.

7. Kopec, B., and I. B. Fritz. 1973. Comparison of properties of carnitine palmitoyl-transferase I with those of carnitine palmitoyltransferase II, and preparation of antibodies to carnitine palmitoyltransferases. J. Biol. Chem. 248: 40694074.

8. McGarry, J. D., and D. W. Foster. 1971. The regulation of ketogenesis from octanoic acid. The role of the tricarboxylic acid cycle and fatty acid synthesis. J. Biol. Chem. 246: 1149-1159.

9. McGarry, J. D., and D. W. Foster. 1971. The regulation 
of ketogenesis from oleic acid and the influence of antiketogenic agents. J. Biol. Chem. 246: 6247-6253.

10. DiMarco, J. P., and C. Hoppel. 1975. Hepatic mitochondrial function in ketogenic states. Diabetes, starvation and after growth hormone administration. J. Clin. Invest. 55: 1237-1244.

11. Norum, K. R. 1964. Palmityl-CoA: carnitine palmityltransferase. Purification from calf-liver mitochondria and some properties of the enzyme. Biochim. Biophys. Acta. 89: 95-108.

12. Lossow, W. J., and I. L. Chaikoff. 1954. Carbohydrate sparing of fatty acid oxidation. I. The relation of fatty acid chain length to the degree of sparing. II. The mechanism by which carbohydrate spares the oxidation of palmitic acid. Arch. Biochem. Biophys. 57: 23-40.
13. McGarry, J. D., P. H. Wright, and D. W. Foster. 1975. Hormonal control of ketogenesis. Rapid activation of hepatic ketogenic capacity in fed rats by anti-insulin serum and glucagon. J. Clin. Invest. 55: 1202-1209.

14. Guynn, R. W., D. Veloso, and R. L. Veech. 1972. The concentration of malonyl-CoA and the control of fatty acid synthesis in vivo. J. Biol. Chem. 247: 7325-7331.

15. Pilkis, S. J., J. P. Riou, and T. H. Claus. 1976. Hormonal control of $\left[{ }^{14} \mathrm{C}\right]$-glucose synthesis from $\left[\mathrm{U}-{ }^{14} \mathrm{C}\right]-$ dihydroxyacetone and glycerol in isolated hepatocytes. J. Biol. Chem. 251: 7841-7852.

16. Cook, G. A., M. R. Lakshmanan, and R. L. Veech. 1977. The effect of glucagon on hepatic malonyl-CoA concentration and on lipid synthesis. Fed. Proc. 36: 672. 